\title{
On possible psychophysical maps: I. Quadratic transformations
}

\author{
PETER H. SCHÖNEMANN \\ Purdue University, West Lafayette, Indiana 47907
}

\begin{abstract}
A certain class of quadratic transformations can be used to map multidimensional physical spaces into multidimensional perceptual spaces; their numerical representations change under various coordinate transformations in both spaces.
\end{abstract}

The problem of reiating two or more multidimensional spaces arises both in scaling and in factor analysis. The technology for fitting such transformations optimally in some sense (usually a least squares sense) is reasonably well understood for the group of affine transformations and its subgroups: similarities, rigid motions, and rotations ("Procrustes" problems, e.g., Ahmavaara, 1957; Borg, 1978; Cliff, 1966; Fischer \& Roppert, 1964; Gower, 1975; Green, 1952; Kristof, 1964; Mosier, 1939; Schönemann, 1964, 1966; Schönemann \& R. M. Carroll, 1970). A defining characteristic of affine transformations is that they carry parallel lines into parallel lines. More recently, a number of psychophysical studies have suggested the need for more general transformations that are not subject to this limitation. This paper summarizes some preliminary results on one type of such more general, "transaffine" mappings.

For a concrete illustration, consider the results of a recent psychophysical study by Krantz and Tversky (1975). They presented 17 rectangles of various shapes and sizes in pairs to a number of subjects. The experimental task was to indicate on a scale of 1-10 how "dissimilar" the two stimuli appeared in each presentation. The main objective of the study was apparently to test ordinally a so-called "decomposability" condition (see Beals, Krantz, \& Tversky, 1968), which is a generalization of the well-known family of Minkowsky metrics. Krantz and Tversky tested it for two pairs of derived physical measures that were defined in terms of the height $(\mathrm{h})$ and width (w) of the rectangles:

$$
\begin{aligned}
& T_{\mathrm{q}_{1}}: \mathrm{E} \rightarrow \mathrm{S}: \mathrm{u}=\log \mathrm{h}, \mathrm{v}=\log \mathrm{w} \\
& \mathrm{T}_{\mathrm{q}_{2}}: \mathrm{E} \rightarrow \mathrm{S}: \mathrm{a}=\mathrm{u}+\mathrm{v}, \mathrm{s}=\mathrm{u}-\mathrm{v},
\end{aligned}
$$

where $\mathrm{E}$ denotes the physical, experimentally controllable space that is represented by a (possibly bounded) region in $\operatorname{Re} \times \mathbf{R e}=\mathbf{R e}^{2}$, and $S \subset R^{2}$ denotes the subjective space. The second pair, a,s, can be interpreted as log transforms of area (hw) and shape (h/w). Krantz and Tversky found decomposability violated for both Equations 1 and 2. This means that neither pair can give rise to Minkowsky metrics monotone with the observed dissimilarities. Krantz and Tversky then processed the observed dissimilarities with two multidimensional scaling programs. They accepted a fit of the INDSCAL model (J. D. Carroll \& Chang, 1970) in two dimensions, but without testing it with any stringency. If the assumptions of the model are met, then the coordinate axes in S, that is, the "psychological dimensions," are uniquely identified by the data. ${ }^{1}$ The main result of this scaling analysis was a distortion of the design configuration that seemed to corroborate the results

I wish to thank the National Institute of Mental Health for Small Grant 1R03MH33388-01, Professor Mike Drazin for many enjoyable hours devoted to the discussion of diverse topics related to this and other projects, and my wife, Roberta, for a great deal of help in typing the manuscript. of the ordinal analysis: Since the stimuli had been defined so that successive levels on both dimensions ( $h$ and $w$ ) corresponded to constant length ratios, the physical design configuration in $\mathrm{E}$ was a square, rotated through $45 \mathrm{deg}$. The square was distorted into a trapezoid in the subjective space S (Krantz \& Tversky, 1975, Figure 1). This means that subjective shape varies as a combined function of physical area and shape.

As Schönemann (1977) pointed out, the results of this scaling analysis, if valid, also imply the definition of a third pair of subjective variables that must satisfy decomposability and must yield euclidean distances monotone with the observed similarities, as a direct consequence of the claimed successful euclidean embedding. The mapping cannot be linear (as Krantz \& Tversky, 1975, implicitly assumed when they investigated Equations 1 and 2), because affine transformations preserve parallelism. Inspection of the two figures shows that the map can be written:

$$
T_{q_{3}}: E \rightarrow S: a^{*}=a, s^{*}=s(1+c a),
$$

where c is a (small) constant. The "starred" quantities are the psychological dimensions expected to reproduce the order of the observed dissimilarities, and the "unstarred" quantities are the (derived) physical variables defined in Equation 2. This mapping reflects the main finding of the ordinal and scaling analysis: Perceived shape increases with area.

In the literature, one finds similar, although of course not identical, maps in other studies of this type. For example, in the experiments reported by Eissler and Roskam (1977) and Roskam (1979), the stimuli were vectors of various lengths and orientation. If they are interpreted as diagonals of rectangles, then the design configuration in the physical space is again square. This design is mapped into a general quadrilateral in the subjective perceptual space. The map from $E$ to $S$ is approximately of the form,

$$
\mathrm{T}_{\mathrm{q}_{4}}: \mathrm{E} \rightarrow \mathrm{S}: \mathrm{u}=\mathrm{ax}+\mathrm{cxy} ; \mathrm{v}=\mathrm{by}+\mathrm{dxy},
$$

where $x$ and $y$ are the independent physical variables, and $u$ and $v$ are the subjective perceptual dimensions.

\section{QUADRATIC TRANSFORMATIONS}

The psychophysical maps, Equations 3 and 4, are special cases of a larger class of transformations that can be written:

$$
T_{q}: E \rightarrow S: y_{k}=\zeta^{\prime} G_{k} \zeta, k=1, q,
$$

where $G_{k}$ is a $(p+1) \times(p+1)$ symmetric matrix, $p$ is the number of independent variables, $q$ is the number of dependent variables, and

$$
\zeta^{\prime}=\left(\xi^{\prime}, 1\right), \text { and } \xi^{\prime}=\left(x_{1}, x_{2}, \ldots, x_{p}\right)
$$

contains the $p$ physical variables $x_{i}(i=1, p)$. 
Since the $y_{k}$ are quadratic functions of the $x_{i}$, such maps will be called "quadratic transformations." 2 The exact type depends on the coordinates $g_{i j k}$ in $G_{k}$. For fixed $y_{k}$, Equation 5 defines $q$ quadrics. On setting some of the elements in $G_{k}$ to zero, or by constraining them in some other way, a number of familiar special cases can be obtained, including translations, rotations, rigid motions, and affine transformations. For the present purpose, the most interesting case arises when the transformation from $E$ to $S$ is truly "transaffine," as it was in Equations 3 and 4.

For what follows, it will be convenient to distinguish between "original" physical variables $\mathrm{x}_{\mathrm{j}}{ }^{*}$, which are those actually used in the expriment, and "derived" physical variables $x_{j}$, which result from the $\mathrm{x}_{\mathrm{j}}{ }^{*}$ by applying some suitably chosen linear transformation $\mathrm{W}$ so that $\mathrm{T}_{\mathrm{q}}$ takes on a simpler, more easily interpreted form. With this notation, Equation 5 can be written, when $\mathrm{p}=\mathrm{q}=2$ :

$$
\begin{gathered}
y_{k}=a_{k_{1}} x_{1}^{*}+a_{k_{2}} x_{2}{ }^{*}+b_{k_{1}} x_{1}{ }^{* 2}+2 c_{k} x_{1}{ }^{*} x_{2}{ }^{*}+b_{k_{2}} x_{2}{ }^{* 2}+d_{k}, \\
(k=1,2)
\end{gathered}
$$

Of particular interest are those cases in which a linear transformation $\xi=W \xi^{*}$ can be found that eliminates the squared terms, so that $b_{k_{1}}=b_{k_{2}}=0$. In this case, $T_{q}$ takes on the simpler form,

$$
y_{k}=\alpha_{k}^{\prime} \xi+\xi^{\prime} Q_{k} \xi+d_{k}, \xi=W \xi^{*}, k=1,2,
$$

where

$$
Q_{k}=\left(q_{i j k}\right), q_{i j k}=c_{k}(0) \text { if } i \neq j(i=j), \alpha_{k}{ }^{\prime}=\left(a_{k_{1}}, a_{k_{2}}\right) \text {, }
$$

and where $W=.\left(w_{i k}\right)$ is the linear transformation that carries the original physical measures, $x_{k}{ }^{*}$, into the derived physical measures, $x_{k}$. For example, in the Krantz and Tversky (1975) study, the $x_{k}^{*}$ are $(\log -)$ height and length and the $x_{k}$ are $(\log -)$ area and shape. Equations 8 and 9 can be written:

$$
\eta=A \xi+2 \gamma \mathbf{x}_{1} \mathbf{x}_{2}+\delta, \xi=W \xi^{*}
$$

with $\eta^{\prime}=\left(\mathrm{y}_{1}, \mathrm{y}_{2}\right), \mathrm{A}=\left(\mathrm{a}_{\mathrm{kj}}\right), \gamma^{\prime}=\left(\mathrm{c}_{1}, \mathrm{c}_{2}\right), \delta^{\prime}=\left(\mathrm{d}_{1}, \mathrm{~d}_{2}\right)$. The rest of this paper will be limited to such planar maps.

\section{GEOMETRIC INTERPRETATION}

The slightly different formulations (Equations 7-10) convey different geometrical aspects of such mappings, which are most easily described in the derived space. On forming the difference $c_{2} y_{1}-c_{1} y_{2}$ in Equation 10, one finds:

$$
c_{2} y_{1}-c_{1} y_{2}=p_{1} x_{1}+p_{2} x_{2}+k_{1},
$$

where

$$
p_{1}=c_{2} a_{11}-c_{1} a_{21}, p_{2}=c_{2} a_{12}-c_{1} a_{22}, k_{1}=c_{2} d_{1}-c_{1} d_{2} \text {. }
$$

Thus $T_{q}$ establishes a 1:1 correspondence between the family of parallel lines $p_{1} x_{1}+p_{2} x_{2}=$ const in $E$, and the family of parallel lines $c_{2} y_{1}-c_{1} y_{2}=$ const in $S$. One further finds from Equation 7 that all lines parallel to the coordinate axes in $\mathrm{E}, \mathrm{x}_{\mathrm{k}}=$ const, map into a family of straight lines with varying slopes and intercepts in $S$. The lines $y_{k}=$ const in $S$ are images of hyperbolae in $E$. In general, lines in $\mathrm{E}$ will map into conics in $\mathrm{S}$. As long as the elements $c_{k}$ in $\gamma$ are "small" relative to the $a_{k j}$ in $A, T_{q}$ will not depart too much from an affine transformation.

The quadratic map, $T_{q}$, is not unrestrictedly invertible. To find the singularities, we set the Jacobian to zero:

$$
\left|\partial y_{k} / \partial \xi\right|=\left|\alpha_{1}+2 Q_{1} \xi, \alpha_{2}+2 Q_{2} \xi\right|=0 \text {. }
$$

On expanding the determinant, one obtains the line,

$$
\mathrm{p}_{1} \mathrm{x}_{1}-\mathrm{p}_{2} \mathrm{x}_{2}+|\mathrm{A}|=0,
$$

as the locus of singular points. This lack of unrestricted invertibility does not vitiate the potential utility of such maps. The main virtues of the $T_{q}$ are ease of computability and interpretation, and the availability of standard statistical tests. As will be shown elsewhere, they can be approximated by other maps that are more easily and unrestrictedly inverted but less easy to fit and to interpret.

\section{STATISTICAL ESTIMATION AND TESTS}

Equation 7 is the expected value of a bivariate curvilinear regression problem. A convenient and optimal method of estimating the parameters of $\mathrm{T}_{\mathrm{q}}$ on the basis of $\mathrm{N}$ replications in $\mathrm{X}^{*}=$ $\left(\mathrm{x}_{\mathrm{ik}}{ }^{*}\right), \mathrm{Y}=\left(\mathrm{y}_{\mathrm{ik}}\right), \mathrm{i}=1, \mathrm{~N}$ is, therefore, provided by the general linear model (GLM):

$$
\mathbf{Y}=\mathbf{X} * \mathbf{M}+\mathbf{U},
$$

where $U=\left(u_{i k}\right)$ is the residual matrix, $M^{\prime}=(A, \gamma, \delta)$ contains the parameters $a_{k j}, b_{k}, c_{k}, d_{k}$, and

$$
\mathrm{X}^{* *}=\left(\mathrm{x}_{\mathrm{i}_{1}}{ }^{*}, \mathrm{x}_{\mathrm{i}_{2}}{ }^{*}, \mathrm{x}_{\mathrm{i}_{1}}^{* 2}, 2 \mathrm{x}_{\mathrm{i}_{1}}^{*} \mathrm{x}_{\mathrm{i}_{2}}{ }^{*}, \mathrm{x}_{\mathrm{i}_{2}}^{* 2}, 1\right)
$$

is the design matrix. For $\mathbf{M}$ to be (statistically) estimable, $\mathrm{X}^{* *}$ must be of full column rank. Under the well-known mild assumptions of the GLM, it is then possible to test linear hypotheses of the form,

$$
H_{0}: Q^{\prime} M P=0 \text {, }
$$

for all conformable full column rank Q,P. In particular,

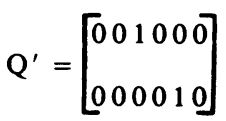

can be used to test the hypothesis that the simpler form, Equation 8 , suffices to describe $T_{q}$. Since the mechanics of the GLM are well documented elsewhere (e.g., Seber, 1966), they need not be repeated here.

A minor complication in practice is the linear transformation, $\xi=W \xi^{*}$, which carries the original physical measures into the derived measures, permitting the simpler representation of $T_{q}$. If the $\mathbf{x}_{\mathbf{k}}$ are physical measures, as assumed, then this transformation is not "admissible" in the sense of measurement theory. Rather, it is an empirically meaningful transformation that affects the outcome of the statistical test. Although some progress has been made in developing an optimal $\mathrm{W}$ for minimizing the contribution of the squared terms in Equation 16, the problem needs further investigation. Fortunately, the exact choice of $\mathrm{W}$ does not appear to be critical. To maximize the fit of the reduced transformation, Equations $8-10, X^{*}$ should be rotated so that corresponding sides of the configuration are approximately parallel. For example, for the Krantz and Tversky (1975) data, ${ }^{3}$ a rotation of $X^{*}$ through $\pi / 2$, as Schönemann $\left(1977\right.$, p. 163) suggested, led to a reduced $T_{q}$ of the form of Equation 8, which explained $99.4 \%$ of the variance of subjective coordinates as quadratic functions of the physical coordinates. Moderate changes in $W$, within $20 \mathrm{deg}$ around $\pi / 2$, did not appreciably affect the $c_{k}$. This relative robustness means, on the other hand, that the derived physical measures, $x_{k}$, are only approximately defined by $W$ in terms of the original $x_{k}{ }^{*}$.

\section{CANONICAL REDUCTIONS IN S}

Since it is assumed that the coordinates $\mathrm{Y}$ in $\mathrm{S}$ result from a distance-based scaling analysis, they are subject to certain 
"admissible transformations," which arbitrarily change the numerical representation of $\mathrm{Y}$, and hence, also of $\mathrm{T}_{\mathrm{q}}$. It is then reasonable to use these admissible transformations to simplify $T_{a}$ as much as possible, so as to obtain a parsimonious and, hopefully, empirically stable and substantively revealing "canonical form" of the transformation $T_{q}$. The extent of possible simplification depends on the scaling model. Occasionally, $Y$ will be determined within a translation and a central dilation. More typically, $Y$ will be determined within rigid motions or similarities. Both Horan's (1969) model and the INDSCAL model leave the stimulus coordinates on two (unrelated) interval scales; that is, they preclude rotations, provided, of course, these models fit (see Footnote 1).

Although it is not possible to treat all details of such identifiability problems here, a few general observations will be made. The first problem to be dealt with is the multidimensional analogue of a question raised by Luce (1959) (see also Rozeboom, 1962): Does the general mathematical form of the mapping $T_{r}$ reproduce itself under all admissible transformations of $\mathrm{X}$ and $\mathrm{Y}$ ? The answer is affirmative for all affine transformations of $\xi$ and $\eta$, since Equation 8 retains its form under affine transformations of $\xi$, and Equation 10 under affine transformations of $\eta$. This is more than needed. If one subjects the coordinates $\mathrm{Y}$ in $\mathrm{S}$ to affine transformations,

$$
\mathrm{T}: \mathrm{S} \rightarrow \mathrm{S}: \eta=\mathrm{R} \eta^{*}+\beta, \mathrm{R} \text { nonsingular, } \beta^{\prime}=\left(\mathrm{b}_{1}, \mathrm{~b}_{2}\right),
$$

then substitution into Equation 10 gives

$$
\eta^{*}=\mathbf{R}^{-1} \mathbf{A} \xi+2 \mathbf{R}^{-1} \gamma \mathbf{x}_{1} \mathbf{x}_{2}+\mathbf{R}^{-1}(\delta-\beta),
$$

which is again of the form of Equation 10. On choosing $\mathbf{R}=\mathbf{A}$, $\delta=\beta$, and writing $\gamma^{*}=R^{-1} \gamma$, one obtains

$$
\eta^{*}=\xi+2 \gamma^{*} x_{1} x_{2}
$$

as a canonical form for $T_{q}$, when the $y_{k}$ are determined within affine transformations. This form has a very simple interpretation: Each physical variable corresponds directly to a subjective variable, and the $c_{k}{ }^{*}$ indicate the extent of the transaffine deformation. The same form can also be used if $\mathrm{Y}$ has been derived from euclidean distances, which define it within rigid motions. The change in $\mathrm{S}$ from $\eta$ to $\eta^{*}=\mathrm{A}^{-1} \eta$ can then be interpreted as a change from an orthogonal to an oblique coordinate system in $S$. To preserve the observed distances, a new metric, $\mathbf{A}^{\prime} \mathbf{A}$, can be introduced, so that

$$
\mathrm{d}^{2}\left(\eta_{\mathrm{i}}, \eta_{\mathrm{j}}\right)=\left(\eta_{\mathrm{i}}^{*}-\eta_{\mathrm{j}}^{*}\right)^{\prime} \mathbf{A}^{\prime} \mathrm{A}\left(\eta_{\mathrm{i}}^{*}-\eta_{\mathrm{j}}^{*}\right)=\left(\eta_{\mathrm{i}}-\eta_{\mathrm{j}}\right)^{\prime}\left(\eta_{\mathrm{i}}-\eta_{\mathrm{j}}\right)
$$

Since only rigid motions are admissible, the new metric $\mathbf{A}^{\prime} \mathbf{A}$ is empirically meaningful in this case. Such oblique transformations have been used in factor analysis to reduce factor patterns to "simple structure." Here, they can be used to simplify mappings.

On the other hand, if it is desired to preserve the original euclidean metric, then the transformations in $S$ will usually be limited to similarities:

$$
\mathrm{T}: \mathrm{S} \rightarrow \mathrm{S}: \eta^{*}=\mathrm{cR} \eta+\beta, \mathrm{R}^{\prime} \mathrm{R}=\mathrm{I}, \mathrm{c}>0 \text { in } \mathbf{R e} .
$$

In this case, a number of options are available for simplifying $T_{q}$ : (1) The singular value decomposition of $A$ can be used to define a unique $R$ that symmetrizes $A$, the linear part of $T_{q}$. (2) $R$ can be used to introduce one zero in $\gamma^{*}$, thus eliminating the mixed term $x_{1} x_{2}$ for one of the subjective variables. (3) $R$ can be used to rotate $\gamma$ into $J=(1,1)^{\prime}$, thus equalizing the contribution of the mixed term to each new subjective variable. (4) $R$ can be used to triangularize $A$, so that either $a_{12}{ }^{*}=0$ or $a_{21}{ }^{*}=0$. The two choices in Options 2 and 4 are equivalent within permutations of the original variables.
Although the choice among the various options for simplifying $\mathrm{T}_{\mathrm{q}}$ under similarities in $\mathrm{S}$ is ultimately a question of empirical utility, Options 2 and 4 seem most attractive at this time. If the physical variables $x_{k}$ are on two unrelated ratio scales, then Option 2 can be written

$$
\begin{aligned}
& y_{1}^{*}=x_{1}+a_{12}{ }^{*} x_{2}+d_{1}^{*} \\
& y_{2}^{*}=a_{21}{ }^{*} x_{1}+x_{2}+2 c_{2}{ }^{*} x_{1} x_{2}+d_{2}{ }^{*} .
\end{aligned}
$$

Under the same assumptions, Option 4 can be written

$$
\begin{aligned}
& y_{1}^{*}=x_{1} \quad+2 c_{1}^{*} x_{1} x_{2}+d_{1}^{*} \\
& y_{2}^{*}=a_{21}^{*} x_{1}+x_{2}+2 c_{2}^{*} x_{1} x_{2}+d_{2}^{*} .
\end{aligned}
$$

If the $x_{k}$ are on a joint ratio scale, only one of the $a_{k k} *$ can be set to unity in Equations 24 and 25.

If the $y_{k}$ are on interval scales, Equation 20 reduces to

$$
\eta^{*}=\mathrm{D}_{\mathrm{p}}^{-1} \xi+2 \mathrm{D}_{\mathrm{p}}^{-1} \gamma^{*} \mathrm{x}_{1} \mathrm{x}_{2}
$$

where the free matrix $D_{p}=\operatorname{diag}\left(p_{1}, p_{2}\right)$ leaves parameters in the same rows of $\left(A^{*}, \gamma^{*}\right)$ on joint ratio scales.

\section{REFERENCE NOTES}

1. Schönemann, P. H. An algebraic solution for a class of subjective metrics model. Paper presented at the meetings of the Psychometric Society, St. Louis, April 1971.

2. Schulz, U. An alternative procedure for the analysis of similarity data and its comparison to the IDIOSCAL and INDSCAL procedure. Paper presented at the International Symposium on Recent Developments in Multidimensional Scaling and Related Areas, Aachen, 1976.

\section{REFERENCES}

AhmavaAra, V. Transformation analysis of factorial data. Annals Academiae Scientiarum Fennicae, 1957, B, 106.

Beals, R., Krantz, D. H., \& Tversky, A. Foundations of multidimensional scaling. Psychological Review, 1968, 75, 127-142.

Borg, I. Procrustean analysis of matrices with different row order. Psychometrika, 1978, 42, 277-278.

Carroll, J. D., \& Chang, J. J. Analysis of individual differences via an N-way generalization of "Eckart-Young" decomposition. Psychometrika, 1970, 35, 283-319.

Cliff, N. Orthogonal rotation to congruence. Psychometrika, $1966,31,33-42$.

Eissler, H., \& Rosкam, E. E. Multidimensional similarity: An experimental and theoretical comparison of vector, distance, and set theoretic models. I. Models and internal consistency of data. Acta Psychologica, 1977, 41, 1-46, 335-363.

Fischer, G., \& Roppert, J. J. Bemerkungen zu einem Verfahren der Transformationsanalyse. Archiv für die gesamte Psychologie, 1964, 116, 98-100.

Gower, J. C. Generalized Procrustes analysis. Psychometrika, $1975,40,33-55$.

Grauste in, W. C. Introduction to higher geometry. New York: Macmillan, 1948.

GreEN, B. F. The orthogonal approximation of an oblique simple structure in factor analysis. Psychometrika, 1952, 17, 429-440.

Horan, C. B. Multidimensional scaling: Combining observations when individuals have different perceptual structures. Psychometrika, 1969, 34, 139-165.

Krantz, D. H., \& Tversky, A. Similarity of rectangles. Journal of Mathematical Psychology, 1975, 12, 4-34. 
KRISTOF, W. Die beste orthogonale Transformation zur gegenseitigen Überführung zweier Faktorenmatrizen. Diagnostica, 1964, 10, 87-90.

Luce, R. D. On the possible psychophysical laws. Psychological Review, 1959, 66, 81-95.

Mosier, C. I. Determining a simple structure when loadings for certain tests are known. Psychometrika, 1939, 4, 149-162.

Rosкам, E. E. Multidimensional scaling by metric transformation of data. In J. C. Lingoes, E. E. Roskam, \& I. Borg (Eds.), Geometric representations of relational data (2nd ed.). Ann Arbor, Mich: Mathesis, 1979.

Roze воом, W. The untenability of Luce's principle. Psychological Review, 1962, 69, 542-547.

Schönemann, P. H. A solution of the orthogonal Procrustes problem with applications to orthogonal and oblique rotation. Unpublished PhD dissertation, University of Illinois, 1964.

Schönemann, P. H. A generalized solution of the orthogonal Procrustes problem. Psychometrika, 1966, 31, 1-10.

Schönemann, P. H. An algebraic solution for a class of subjective metrics models. Psychometrika, 1972, 37, 441-451.

SchönemanN, P. H. Note. Similarity of rectangles. Journal of Mathematical Psychology, 1977, 16, 161-165.

Schönemann, P. H., \& Carroll, R. M. Fitting one matrix to another under choice of a central dilation and a rigid motion. Psychometrika, 1970, 35, 245-255.

Schönemann, P. H., James, W. L., \& Carter, F. S. Statistical inference in multidimensional scaiing: A method for fitting and testing Horan's model. In J. C. Lingoes, E. E. Roskam, \& I. Borg (Eds.), Geometric representations of relational data (2nd ed.). Ann Arbor, Mich: Mathesis, 1979.

Schulz, U. Über zwei Modelle der multidimensionalen Skalierung unter Berucksichtigung individueller Differenzen. Unpublished PhD dissertation, University of Marburg, 1971.

Seber, G. A. F. The linear hypothesis: A general theory. New York: Hafner, 1966.

\section{NOTES}

1. At the time their work was done, there apparently existed no practical tool for falsifying the critical (diagonality) assumption on which this claim rests. Such a tool has now been made available (Schönemann, James, \& Carter, 1979). That the model proposed by Horan (1969) and the variant recommended by Carroll and Chang (1970) are falsifiable in principle had been shown by Schönemann (1972, Note 1) and Schulz (1971, Note 2).

2. This terminology is not standard. Older texts, for example, Graustein (1948, p. 403), use the term "quadratic transformations" for certain birational transformations.

3. Due to space limitations, detailed numerical graphical illustrations are not presented in this paper.

(Received for publication July 16, 1979.) 